\title{
Decrypting the H-NS-dependent regulatory cascade of acid stress resistance in Escherichia coli
}

Evelyne Krin ${ }^{1 *}$, Antoine Danchin², Olga Soutourina ${ }^{3,4}$

\begin{abstract}
Background: H-NS regulates the acid stress resistance. The present study aimed to characterize the H-NSdependent cascade governing the acid stress resistance pathways and to define the interplay between the different regulators.

Results: We combined mutational, phenotypic and gene expression analyses, to unravel the regulatory hierarchy in acid resistance involving H-NS, RcsB-P/GadE complex, HdfR, CadC, AdiY regulators, and DNA-binding assays to separate direct effects from indirect ones. RcsB-P/GadE regulatory complex, the general direct regulator of glutamate-, arginine- and lysine-dependent acid resistance pathways plays a central role in the regulatory cascade. However, H-NS also directly controls specific regulators of these pathways (e.g. cadC) and genes involved in general stress resistance (hdeAB, hdeD, dps, adiY). Finally, we found that in addition to H-NS and RcsB, a third regulator, HdfR, inversely controls glutamate-dependent acid resistance pathway and motility.

Conclusions: H-NS lies near the top of the hierarchy orchestrating acid response centred on RcsB-P/GadE regulatory complex, the general direct regulator of glutamate-, arginine- and lysine-dependent acid resistance pathways.
\end{abstract}

\section{Background}

In Escherichia coli, complex cellular responses are controlled by networks of transcriptional factors that regulate the expression of a diverse set of target genes, at various hierarchical levels. H-NS, a nucleoid-associated protein, is a top level regulator affecting the expression of at least 250 genes, mainly related to the bacterial response to environmental changes [1]. Among its various targets, it regulates in opposite directions the flagella-dependent motility and the acid stress resistance [1]; the first via the control of flhDC master flagellar operon by acting both directly and indirectly via regulators HdfR and RcsB [2-6]; the second by repressing the genes involved in three amino acid decarboxylase systems, dependent on glutamate, lysine and arginine, via the RcsB-P/GadE regulatory complex [6]. In this regulatory process $\mathrm{H}-\mathrm{NS}$ represses the expression of gadE (encoding the central activator of the glutamate-dependent acid resistance pathway) both in a direct and an indirect way, via EvgA, YdeO, GadX and GadW $[1,7,8]$, while it decreases $r c s D$ expression, essential

\footnotetext{
* Correspondence: ekrin@pasteur.fr

'Unité de Plasticité du Génome Bactérien, Institut Pasteur, France Full list of author information is available at the end of the article
}

to the phosphorylation of RcsB (the capsular synthesis regulator component) required for the formation of the regulatory complex with GadE [6]. In the glutamate pathway, the RcsB-P/GadE regulatory complex controls the expression of two glutamate decarboxylase paralogues GadA and GadB, the glutamate/gamma-aminobutyrate antiporter GadC, two glutamate synthase subunits GltB and GltD, the acid stress chaperones HdeA and HdeB, the membrane protein $\mathrm{HdeD}$, the transcriptional regulator YhiF (DctR) and the outer membrane protein Slp [6]. The complex also induces an arginine decarboxylase, AdiA, and an arginine:agmatine antiporter, $\mathrm{AdiC}(\mathrm{YjdE})$, essential for arginine-dependent acid resistance. Finally, the complex regulates a lysine decarboxylase, $\mathrm{CadA}$, and a cadaverine/ lysine antiporter, $\mathrm{CadB}$, essential for lysine-dependent acid resistance $[1,6,9]$. Apart from the $\operatorname{gadBC}$ operon, the most important genes involved in acid resistance are present within the acid fitness island (AFI), a $15 \mathrm{~kb}$ region both repressed by H-NS and under the control of RpoS [10,11]. Recent global chromatin immunoprecipitation studies revealed that H-NS binds to several loci within this region, including hdeABD $[12,13]$. However, neither AdiY, the main regulator of the arginine-dependent response that 
controls adiA and adiC expression [14,15] nor CadC, the main regulator of lysine-dependent response controlling $\operatorname{cadBA}$ [16], were yet found among the identified H-NS targets.

In the present study, we aimed at further characterizing the H-NS-dependent cascade governing acid stress resistance pathways to identify the missing intermediary regulator(s) or functional protein(s) controlled by H-NS and to define the interplay between the different regulators and their targets.

\section{Methods}

\section{Bacterial strains and plasmids}

Bacterial strains and plasmids used in this study are listed in Table 1. Mutants were constructed by replacing the entire gene of interest with an antibiotic cassette using the CF10230 strain, as previously described [17]. These mutations, as well as their Miki and Keio collection counterparts from NBRP (NIG, Japan): E. coli $[18,19]$ were subsequently transduced into FB8 hns::Sm derivative strains, using P1vir phage. When required, antibiotics were added: ampicillin $\left(100 \mu \mathrm{g} \mathrm{ml}{ }^{-1}\right)$, streptomycin $\left(10 \mu \mathrm{g} \mathrm{ml}^{-1}\right)$, kanamycin $\left(40 \mu \mathrm{g} \mathrm{ml}^{-1}\right)$, tetracycline $\left(15 \mu \mathrm{g} \mathrm{ml}^{-1}\right)$.

\section{Resistance to low $\mathrm{pH}$}

The experiment was performed at least twice, as previously described [6].

\section{RNA preparation and Real-time quantitative RT-PCR}

The experiment was performed twice, as previously described [6]. Primers used in real-time quantitative RTPCR experiments are listed in Additional file 1.

\section{Protein purification}

H-NS-His6 was purified as previously described [20]. Recombinant proteins HdfR-His6, His6-RcsB ${ }_{\mathrm{D} 56 \mathrm{E}}$, GadEHis6 and Strep-AdiY were purified as previously described [6].

\section{Gel mobility shift assays}

Gel shift assays were performed with $0.1 \mathrm{ng}\left[\gamma^{32} \mathrm{P}\right]$ labelled probe DNA with purified HdfR-His6, His6$\mathrm{RcsB}_{\mathrm{D} 56 \mathrm{E}}$ (mimicking phosphorylated and activated

Table 1 Bacterial strains and plasmids used in this study

\begin{tabular}{|c|c|c|}
\hline Strain or plasmid & Genotype or description & Reference or source \\
\hline \multicolumn{3}{|l|}{ Strains } \\
\hline JD21162 & KP7600 (F-laclQ lacZdeltaM15 galK2 galT22 lambda- in (rrnD-rrnE)1, W3110 derivative) ydeP ::Km & [19] \\
\hline JD24946 & KP7600 (F- laclQ lacZdeltaM15 galK2 galT22 lambda- in (rrnD-rrnE)1, W3110 derivative) yhiM ::Km & [19] \\
\hline JD25275 & KP7600 (F-laclQ lacZdeltaM15 galK2 galT22 lambda- in (rrnD-rrnE)1, W3110 derivative) hdeA :Km & [19] \\
\hline JD26576 & KP7600 (F- laclQ lacZdeltaM15 galK2 galT22 lambda- in (rrnD-rrnE)1, W3110 derivative) ydeO::Km & [19] \\
\hline JD27509 & KP7600 (F- laclQ lacZdeltaM15 galK2 galT22 lambda- in (rrnD-rrnE)1, W3110 derivative) dps ::Km & [19] \\
\hline JW5594 & BW25113 (rrnB $\triangle a a c Z 4787$ HsdR514 $\triangle(a r a B A D) 567 \Delta(r h a B A D) 568$ rph-1) $\triangle a s I B ~:: K m$ & {$[18]$} \\
\hline JW2366 & BW25113 (rrnB $\triangle a c Z 4787$ HsdR514 $\triangle($ araBAD)567 $\triangle(r h a B A D) 568$ rph-1) $\triangle$ evgA ::Km & [18] \\
\hline EP247 & W3110 cadC1::Tn10 & [41] \\
\hline FB8 & Wild type & [42] \\
\hline BE1411 & FB8 hns::Sm & [43] \\
\hline BE2823 & FB8 hns::Sm $\triangle r c s B:: K m$ & {$[6]$} \\
\hline BE2825 & FB8 hns::Sm $\triangle$ hdfR $\because: T e t$ & This study \\
\hline BE2826 & FB8 hns::Sm dps ::Km & FB8 hns::Sm × P1 JD27509 \\
\hline BE2827 & FB8 hns::Sm rpos $359:: \mathrm{Km}$ & This study \\
\hline BE2828 & FB8 hns::Sm yhiM ::Km & FB8 hns::Sm × P1 JD24946 \\
\hline BE2829 & FB8 hns::Sm $\triangle$ evgA ::Km & FB8 hns::Sm × P1 JW2366 \\
\hline BE2830 & FB8 hns::Sm $\triangle a s l B:: K m$ & FB8 hns::Sm $\times$ P1 JW3772 \\
\hline BE2831 & FB8 hns::Sm ydeP ::Km & FB8 hns::Sm $\times$ P1 JD21162 \\
\hline BE2832 & FB8 hns::Sm ydeO ::Km & FB8 hns::Sm $\times$ P1 JD26576 \\
\hline $\mathrm{BE} 2836$ & FB8 hns:::Sm $\Delta h d e A:: K m$ & FB8 hns::Sm $\times$ P1 JD25275 \\
\hline BE2837 & FB8 hns::Sm $\Delta a d i Y ~:: T e t$ & This study \\
\hline BE2939 & FB8 hns::Sm cadC1::Tn10 & FB8 hns:::Sm $\times$ P1 EP247 \\
\hline \multicolumn{3}{|l|}{ Plasmids } \\
\hline pDIA640 & pet22b :.hdfR with C terminal His tag & This study \\
\hline pDIA642 & pet16b : :rCs $B_{D 56 E}$ with $\mathrm{N}$ terminal His tag & {$[6]$} \\
\hline pDIA645 & pet22b : :gadE with C terminal His tag & {$[6]$} \\
\hline pDIA646 & pet16b ::adiY with $N$ terminal strep tag & This study \\
\hline
\end{tabular}


Table 2 Glutamate-dependent acid resistance of $E$. coli strains

\begin{tabular}{|c|c|}
\hline Strain (relevant genotype) & Glutamate-dependent acid resistance (\% survival) \\
\hline FB8 (wild-type) & 0.1 \\
\hline BE1411 (hns::Sm) & 51.7 \\
\hline BE2823 (hns::Sm $\Delta r c s B)$ & $<0.001$ \\
\hline BE2825 (hns:::Sm $\Delta h d f R$ ) & 12.5 \\
\hline BE2826 (hns::Sm dps::Km) & 20.1 \\
\hline BE2827 (hns::Sm rpos) & 27.5 \\
\hline BE2828 (hns::Sm yhiM::Km) & 24.2 \\
\hline BE2829 (hns::Sm \evgA) & 32.0 \\
\hline BE2831 (hns::Sm ydeP::Km) & 35.6 \\
\hline BE2832 (hns::Sm ydeO::Km) & 38.2 \\
\hline BE2830 (hns::Sm $\triangle a s / B)$ & 38.6 \\
\hline BE2837 (hns:::Sm \adiY) & 5.4 \\
\hline BE2939 (hns::Sm cadC1::Tn10) & 58.1 \\
\hline
\end{tabular}

Data are the mean values of two independent experiments that differed by less than $20 \%$.

RcsB), GadE-His6 and Strep-AdiY proteins as previously described $[6,10]$. For competitive gel mobility shift assays with purified H-NS protein 100-200 ng PCR fragments of target promoter regions and 270-200 ng of competitor DNA fragments, obtained by digestion of pBR322 plasmid with TaqI and $S s p$ I restriction enzymes, were incubated for $15 \mathrm{~min}$ at room temperature with $\mathrm{H}$ NS in the previously described reaction mixture [21]. Protein-DNA complexes were resolved on $3 \%$ or $4 \%$ MetaPhor agarose gel. Primers used in gel mobility shift assays are listed in Additional file 2.

\section{Results}

Determination of new H-NS targets involved in the regulation of glutamate-dependent acid resistance

As H-NS strongly represses the glutamate-dependent acid stress response, there is a very low level of survival after acid stress in the FB8 wild-type context [6]. As a consequence, $\mathrm{H}-\mathrm{NS}$ targets involved in this process are only expressed when hns is removed. To find further H-NSdependent intermediary actors of glutamate-dependent acid resistance, several of the H-NS induced targets, identified in a previous transcriptome analysis [1] and related either to acid stress resistance or to information pathways, were deleted in an hns-deficient strain. We looked for a decreased glutamate-dependent acid resistance, in comparison to that displayed in the parent hns-deficient strain. Different extent of decrease in resistance to acidic conditions was observed with deletion of several genes known to be related to acid stress response including $d p s$ (coding for the Dps protein - DNA-binding protein of starved cells), rpoS (coding for the RNA polymerase sigma-38 factor), yhiM (coding for an inner membrane protein), evgA (coding for a transcriptional activator), $y d e P$ (coding for a putative anaerobic dehydrogenase) and $y d e O$ (coding for a transcriptional regulator, which is a target of sRNA OxyS) (Table 2), suggesting a role in the H-NS-controlled glutamate-dependent acid resistance. Furthermore, a reduced resistance was also observed with genes, not previously associated with acid stress, such as $a s l B$ (coding for an anaerobic sulfatase-maturating enzyme homolog) and $h d f R$ (coding for the H-NS-dependent flhDC regulator) (Table 2). However, the single deletion of several genes including evgA, $y d e P, y d e O$ and $a s l B$ in hns background only slightly affected the acid stress survival, suggesting their redundant function in this H-NS-dependent process.

Determination of H-NS targets involved in arginine and/ or lysine-dependent acid resistance

We wondered whether the new genes identified as H-NScontrolled, but unrelated to regulation by the RcsB-P/ GadE complex, also play a role in the arginine and lysine-

Table 3 Arginine and lysine-dependent acid resistance of E. coli strains

\begin{tabular}{|c|c|c|}
\hline $\begin{array}{l}\text { Strain (relevant } \\
\text { genotype) }\end{array}$ & $\begin{array}{l}\text { Arginine-dependent } \\
\text { acid } \\
\text { resistance (\% } \\
\text { survival) }\end{array}$ & $\begin{array}{l}\text { Lysine-dependent } \\
\text { acid } \\
\text { resistance (\% } \\
\text { survival) }\end{array}$ \\
\hline FB8 (wild-type) & 0.23 & 0.05 \\
\hline BE1411 (hns::.Sm) & 24.50 & 7.64 \\
\hline BE2823 (hns::Sm $\Delta$ rcsB) & 4.44 & 1.00 \\
\hline BE2826 (hns::Sm $\triangle d p s)$ & 0.11 & 0.28 \\
\hline BE2836 (hns::Sm VhdeA) & 5.11 & 5.37 \\
\hline 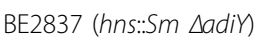 & 1.80 & 7.30 \\
\hline $\begin{array}{l}\text { BE2939 (hns::Sm cadC1:: } \\
\text { Tn10) }\end{array}$ & 24.24 & 0.001 \\
\hline
\end{tabular}

Percentage survival is calculated as $100 \times$ number of c.f.u. per $\mathrm{ml}$ remaining after 2 hours low $\mathrm{pH}$ treatment in the presence of arginine or lysine, divided by the initial c.f.u. per $\mathrm{ml}$ at time zero. Data are the mean values of two independent experiments that differed by less than $15 \%$. 
dependent acid resistance pathways. We performed acid stress assays in the presence of these amino acids with hns-deficient strains also deleted in these genes. Only the deletion of $d p s$ led to dramatically low survival rate in the presence of arginine and lysine, while the deletion of $h d e A$ resulted in a 5 -fold decreased survival rate in the presence of arginine and slightly modified survival rate in the presence of lysine (Table 3). Although the arginine and lysine-dependent acid resistance pathways are regulated by H-NS [1], it is not known whether AdiY and $\mathrm{CadC}$, the specific regulators of these pathways respectively, are controlled by H-NS. Real-time quantitative RT-PCR experiments were carried out on adi $Y$ and $c a d C$ with RNA isolated from FB8 wild-type and hns-deficient strains. We observed that the adiY and cadC RNA level increased five-fold in the hns mutant (Table 4), suggesting that they may mediate the effect of $\mathrm{H}-\mathrm{NS}$ on arginine and lysine-dependent acid stress resistance. To further investigate the role of $a d i Y$ and $c a d C$ in the H-NS-dependent control of acid resistance, each gene was deleted in an hns background and the acid resistance assays were performed in the presence of arginine, glutamate and lysine. In the absence of adi $Y$, much fewer bacteria survived in the presence of glutamate and arginine, but not in the presence of lysine, while the $c a d C$ deletion led to extreme acid stress sensitivity only in the presence of lysine (Table 2 and 3). This suggests a role of CadC regulator in the $\mathrm{H}-\mathrm{NS}$ regulation of the lysine-dependent acid stress resistance and a role of AdiY regulator in the arginine- and glutamate-dependent pathways.

\section{Identification of the target genes for major regulators}

To decipher the regulatory hierarchy in acid stress resistance involving several new $\mathrm{H}-\mathrm{NS}$ controlled regulators, the mRNA level of target genes was compared between wild-type and hns, hns rcsB, hns gadE, hns hdfR, hns adiY mutant strains, using real-time quantitative RT-PCR (Table 4). In particular, we compared the expression ratio between a double mutant and the wild-type strain with that for hns-deficient and the wild-type strain. H-NS having negative effect on target genes, these genes are strongly derepressed in hns mutant in comparison with wild-type strain. If this strong $\mathrm{H}-\mathrm{NS}$ repressive effect is abolished in the absence of a regulator negatively controlled by H-NS, we can conclude that this deleted regulator has positive effect on target gene expression and may be an intermediary actor in H-NS-dependent control for this target, as previously shown [6].

It was found that RcsB and GadE upregulate, at the similar level, newly identified genes involved in acid stress resistance pathways dependent on glutamate $(y h i M$ and $a s(B)$, but these two regulators did not affect the expression of regulatory genes, $c a d C$ and $\operatorname{adi} Y$ (Table 4). Neither RcsB nor GadE controlled $h d f R$ regulatory gene expression (data not shown), suggesting that the $h d f R$ is not the target of RcsB-P/GadE complex. We found that HdfR controlled only the expression of $a s l B$ and gltBD in the glutamatedependent acid stress resistance regulon (Table 4). As expected, AdiY strongly affected $\operatorname{adiA}$ and adiC expression, and also the expression of some genes related to the glutamate specific pathway ( $a s l B, \operatorname{gad} A, \operatorname{gad} B C, \operatorname{glt} B D$, and

Table 4 Quantitative RT-PCR analysis on H-NS targets involved in acid stress resistance

\begin{tabular}{|c|c|c|c|c|c|}
\hline \multirow[b]{2}{*}{ Gene } & \multicolumn{5}{|c|}{ Expression ratio } \\
\hline & hns/wild-type & hns gadE/wild-type & hns $r c s B$ /wild-type & hns hdfR/wild-type & hns adiY/wild-type \\
\hline \multicolumn{6}{|c|}{ Glutamate-dependent specific pathway } \\
\hline $\operatorname{gad} A^{1}$ & 137.21 & nd & $\mathrm{Nd}$ & 150.93 & 41.31 \\
\hline$d c t R^{1}$ & 34.66 & nd & $\mathrm{Nd}$ & 34.32 & 8.84 \\
\hline yhiM & 10.75 & 3.41 & 3.40 & 10.90 & 11.36 \\
\hline$a s / B$ & 12.92 & 0.66 & 1.10 & 0.69 & 1.32 \\
\hline$g \mid t D^{1}$ & 1.68 & nd & $\mathrm{Nd}$ & 0.48 & 0.52 \\
\hline \multicolumn{6}{|c|}{ Arginine-dependent specific pathway } \\
\hline adiA & 16.89 & nd & $\mathrm{Nd}$ & nd & 0.70 \\
\hline adic & 11.62 & nd & $\mathrm{Nd}$ & nd & 1.41 \\
\hline \multicolumn{6}{|c|}{ Lysine-dependent specific pathway } \\
\hline $\operatorname{cadC}$ & 4.62 & 5.77 & 6.38 & nd & nd \\
\hline \multicolumn{6}{|c|}{ General acid stress resistance pathway } \\
\hline hdeA ${ }^{1}$ & 32.37 & nd & $\mathrm{Nd}$ & 41.20 & 6.55 \\
\hline hdeD & 18.96 & nd & $\mathrm{Nd}$ & 17.57 & 5.89 \\
\hline adiY & 5.08 & 5.00 & 5.00 & nd & nd \\
\hline
\end{tabular}

nd: non-determined.

1: Since several genes are organized in operon and/or are highly homologous to each other, results obtained with gadA also corresponds to gadBC; with gltD to gltB; with hdeA to $h d e B$; with $d c t R$ to $s / p$.

Quantitative RT-PCR were performed on total RNA isolated from exponential growth phase cultures. Standard deviations were less than $20 \%$ of the mean. 
slp-dctR) and to general acid resistance ( $h d e A B$ and $h d e D$ ) (Table 4). These results demonstrated a multiple control of several target genes involving different regulators acting independently from each other.

\section{Identification of the new targets directly controlled by RcsB-P/GadE complex}

Gel mobility shift assays were performed with a mixture of purified $\operatorname{RcsB}_{\mathrm{D} 56 \mathrm{E}}$ and GadE proteins to know whether the regulatory complex directly controlled $y$ hiM and $a s l B$. It was established that the $\mathrm{RcsB}_{\mathrm{D} 56 \mathrm{E}} / \mathrm{GadE}$ regulatory complex binds to the promoter regions of the two genes (Figure 1A), demonstrating the direct control by the RcsB-P/GadE complex.

\section{Identification of the targets directly controlled by HdfR or AdiY}

Real-time quantitative RT-PCR analysis showed that HdfR regulates $a s l B$ and $g l t B D$, while AdiY regulates several genes involved in acid stress resistance (adiA, adiC, aslB, gadA, gadBC, gltBD, hdeAB, hdeD and slp$\operatorname{dctR}$ ) (Table 4). To establish whether these regulators control the expression of these genes by direct binding to their promoter regions, gel mobility shift assays were performed with purified HdfR and AdiY proteins. It was found that HdfR binds to the promoter region of $g l t B D$ and that AdiY binds to the promoter regions of $g l t B D$,
adiA and gadABC (Figure 1B). However, no band shift was observed even with higher concentration of regulator with HdfR on the promoter region of $a s l B$ and with AdiY on the promoter regions of adiC, aslB, hdeABD and slp-dctR (Figure 1B), suggesting an indirect regulation for these genes.

\section{Identification of the targets directly controlled by H-NS}

$\mathrm{H}-\mathrm{NS}$ modulates the expression of several regulators controlling acid stress resistance including HdfR, RcsD, EvgA, YdeO, YdeP, GadE, GadW, GadX, AdiY and CadC. However, the direct control by H-NS has not yet been established for the majority of these regulators, except for GadX [22] and HdfR [3]. Furthermore, slp-dctR and $y$ hiM could also be directly repressed by H-NS, as deletion of their regulators, RcsB-P/GadE complex and/ or AdiY, in hns-deficient strain was not sufficient to restore their wild-type mRNA level (Table 4) [6]. Competitive gel mobility shift assays were performed with purified H-NS protein on PCR fragments, corresponding to assayed promoters, and restriction fragments derived from the pBR322 plasmid, used as negative competitors for binding to H-NS protein except for one 217-bp DNA fragment corresponding to the bla promoter used as positive internal control [21]. A preferential binding of $\mathrm{H}-\mathrm{NS}$ was observed to the promoter regions of adiY, cadBA, cadC, evgA, gadE, gadW, hdfR, rcsD, slp-dctR,
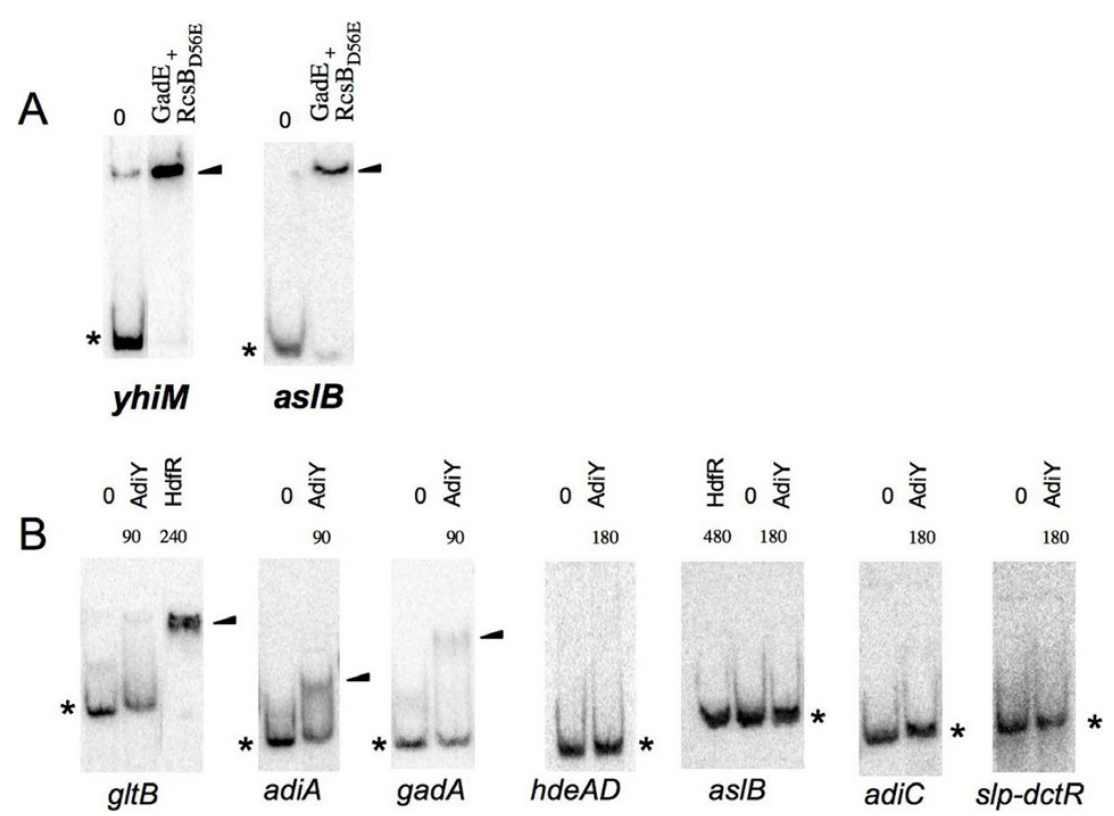

Figure $1 \mathrm{Gel}$ mobility shift assays with GadE/RcsB ${ }_{\mathrm{D} 56 \mathrm{E}}$ complex, HdfR and AdiY. A. Gel mobility shift assays with GadE/RcsB $B_{\mathrm{D} 56 \mathrm{E}}$ COmplex and new DNA targets. Proteins were incubated with DNA targets during $30 \mathrm{~min}$ at $25^{\circ} \mathrm{C}$ in the final reaction mixture volume of $15 \mu \mathrm{l}$. $900 \mathrm{ng}$ of each GadE and RcsB $B_{\text {D5E }}$ protein are used for yhiM and as/B. B. Gel mobility shift assays with HdfR or AdiY proteins. Quantities of purified HdfR or AdiY proteins are indicated above each lane (in $\mathrm{ng}$ ). Gel mobility shift assays (A and B) were performed with $0.1 \mathrm{ng}\left[\gamma^{32} \mathrm{P}\right]$-labelled DNA fragment and loaded on a $6 \%$ polyacrylamide native gel. An arrow points out the position of the DNA-regulatory protein complex. An asterisk marks the position of the unbound probe. 


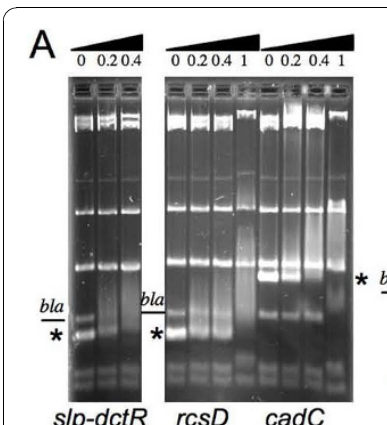

$s / p-d c t R \quad r c s D \quad \operatorname{cad} C$
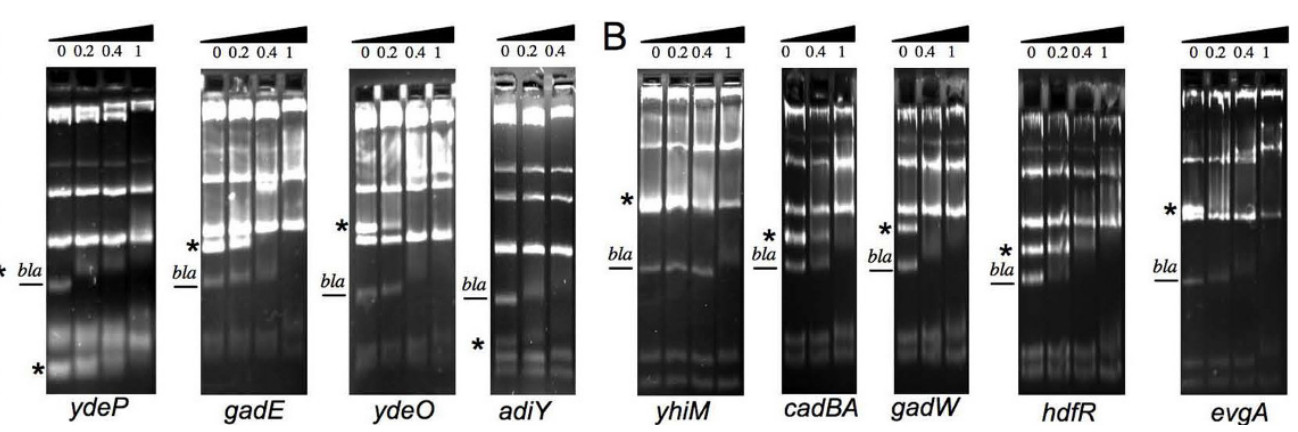

Figure 2 Competitive gel mobility shift assay with H-NS, target promoter fragments and restriction fragments derived from plasmid pBR322. The cleaved plasmid and promoter fragments were incubated with the indicated concentrations of purified $\mathrm{H}$-NS protein (in $\mu \mathrm{M}$ ). After protein-DNA complex formation, the fragments were resolved on a 3\% (A) or 4\% (B) MetaPhor agarose gel. An asterisk indicates the position of the target promoter fragments. "bla" indicates the bla promoter (positive control), the other fragments of plasmid DNA correspond to negative controls. The specific binding of H-NS is observed when bands corresponding to bla and target promoter disappear with increasing concentration of H-NS, the H-NS-DNA complex being difficult to visualize under these conditions.

ydeO, ydeP, yhiM, confirming the direct control by $\mathrm{H}-\mathrm{NS}$ of these genes (Figure 2).

\section{Discussion}

$\mathrm{H}-\mathrm{NS}$ regulates directly and indirectly the $\mathrm{RcsB}-\mathrm{P} / \mathrm{GadE}$ complex, that is located at the centre of the acid resistance network as well as control of motility (Figure 3 ). Furthermore, H-NS modulates the level of several regulatory proteins, unrelated to this complex (e.g. CadC, AdiY, HdfR) (Table 4 and Figure 2) [3]. Among them, only HdfR was previously known as a H-NS target [3]. The present study revealed that, in addition to its role in motility control, HdfR regulates the glutamate-dependent acid resistance pathway, directly inducing glt $B D$ and indirectly controlling aslB (Table 4 and Figure 1, 3). All the results presented in this work were integrated together with previously published data, to propose a model of the complex H-NS-dependent regulatory network governing motility and acid stress resistance processes in E. coli (Figure 3). The new characterized H-NS targets, CadC and AdiY, have no effect on motility (data not shown) and are involved in the H-NS-dependent regulation of lysine and arginine-dependent response to acid stress, respectively (Table 3). Furthermore, we found that AdiY is also involved in glutamate-dependent response to acid stress (Table 2). It directly or indirectly regulates several genes specific to this response including $a s l B$, gltBD, gadA, gadBC, slp-dctR or having more global role in acid stress resistance such as $h d e A B$ and $h d e D$ (Table 4). Interestingly, we demonstrated that H-NS has a direct control effect on the $c a d B A$ promoter (Figure 2), in accordance with the previous suggestion of a competition between the CadC activator and $\mathrm{H}-\mathrm{NS}$ for binding to this promoter region [23]. In addition to its role in the repression of major regulators at high levels of the hierarchy, we have shown that H-NS is able to directly affect acid stress circuits repressing the transcription of several structural genes (e.g. yhiM, slp, dctR) (Figure 2). This is in agreement with the proposed competition between activation by specific regulators and repression by $\mathrm{H}-\mathrm{NS}$, in several bacterial systems $[24,25]$. The results of present study point out the essential role for several intermediary players within H-NS-dependent regulatory network and suggest an accessory role for other regulators in acid stress response. Indeed, the EvgA-YdeO regulatory pathway plays a secondary modulator role in the glutamatedependent acid stress response, in comparison to H-NS. In the same means, AslB and YdeP, two anaerobic enzymes, may have a redundant function in this stress response.

Among the H-NS-regulated genes, we showed that the acid stress chaperones HdeA and $\mathrm{HdeB}$ that solubilized periplasmic protein aggregates at acid $\mathrm{pH}$ [26] are involved in all three pathways of acid stress response. However, their impact is low in the arginineand lysine-dependent pathways (Table 3), while they are essential in the glutamate-dependent pathway [27]. This could be explained by the fact that arginine and lysine amino acids are able to strongly oppose protein aggregation [28]. By contrast, we found that the expression of the $d p s$ gene, directly regulated by H-NS and known to protect cells against multiple stresses [29], is essential to lysine- and arginine-dependent responses to acid stress, while its role is less important during the glutamate-dependent response (Table 2 and $3)$. This implies that the induced glutamate-dependent response provides sufficient cell protection, restricting Dps to a marginal role. This is consistent with the observation that glutamate is widely distributed amino acid representing approximately $15-45 \%$ in the dietary protein content and plays a key physiological role in gastrointestinal tract [30]. 


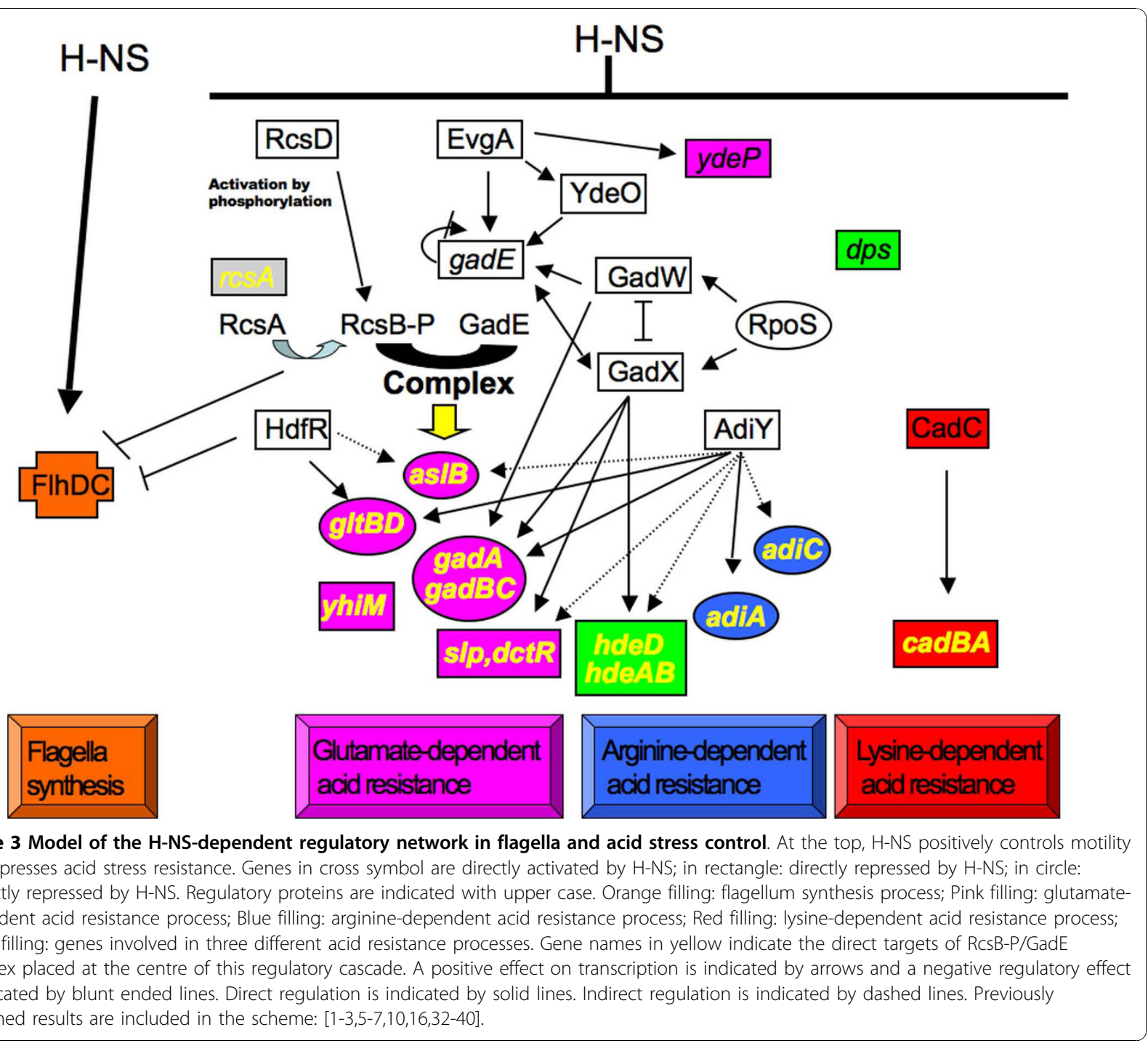

Within this frame of thought, the glutamate decarboxylase system would be the most efficient acid resistance mechanism [31]. This could also explain why three regulators $\mathrm{H}-\mathrm{NS}$, HdfR and RcsB are directly involved in the control of both glutamate-dependent acid stress response and the flagellum biosynthesis. Indeed, as flagellum is a high consumer of ATP and leads to proton entrance during its motor functioning, it is necessary to stop this process to limit cytoplasmic acidification in bacteria and to redirect energy to mechanisms of resistance to stress. Furthermore, the flagellar filaments bear strong antigenic properties in contact with host. We can suppose that these complex high-organized regulations allow a faster adaptation to environmental variations and an increase in survival under adverse conditions found in gastric environment contributing to the virulence of gastrointestinal bacteria, examplified by enteroinvasive E. coli $\mathrm{HN} 280$ [32].

\section{Conclusion}

In $E$. coli, the control of acid stress resistance is achieved by the concerted efforts of multiple regulators and overlapping systems, most of the genes directly involved in acid resistance being both controlled by RcsB-P/GadE complex and by at least one other regulator such as H-NS, HdfR, CadC or AdiY.

\section{Additional material}

Additional File 1: List of primers used in real-time quantitative RTPCR experiments

Additional File 2: List of primers used for gels retardation assay.

\section{Acknowledgements}

We thank Nathalie Sassoon for help in protein purifications and Zeynep Baharoglu for critical reading of the manuscript. Financial support came 
from the Institut Pasteur, the Centre National de la Recherche Scientifique (URA 2171) and the Probactys NEST European programme, grant CT-2006029104. OS is assistant professor at the Universite Paris 7.

\section{Author details}

'Unité de Plasticité du Génome Bactérien, Institut Pasteur, France. ${ }^{2}$ AMAbiotics 5, rue Henri Desbruères, 91030 Evry Cedex, France. ${ }^{3}$ Unité des Bactéries Anaérobies et Toxines, Institut Pasteur, France. ${ }^{4}$ Université Paris 7Denis Diderot, 75205 Paris, France.

\section{Authors' contributions}

EK conceived the study, performed all experiments and drafted the manuscript. $A D$ helped to finalize the manuscript and to place it in perspective, OS helped to analyse the data and to draft the manuscript. All authors read and approved the final manuscript.

Received: 2 June 2010 Accepted: 29 October 2010

Published: 29 October 2010

\section{References}

1. Hommais F, Krin E, Laurent-Winter C, Soutourina O, Malpertuy A, Le Caer JP, Danchin A, Bertin P: Large-scale monitoring of pleiotropic regulation of gene expression by the prokaryotic nucleoid-associated protein, $\mathrm{H}-\mathrm{NS}$. Mol Microbiol 2001, 40(1):20-36

2. Francez-Charlot A, Laugel B, Van Gemert A, Dubarry N, Wiorowski F, Castanie-Cornet MP, Gutierrez C, Cam K: RcsCDB His-Asp phosphorelay system negatively regulates the flhDC operon in Escherichia coli. Mol Microbiol 2003, 49(3):823-832.

3. Ko M, Park C: H-NS-Dependent regulation of flagellar synthesis is mediated by a LysR family protein. J Bacteriol 2000, 182(16):4670-4672.

4. Soutourina O, Kolb A, Krin E, Laurent-Winter C, Rimsky S, Danchin A, Bertin P: Multiple control of flagellum biosynthesis in Escherichia coli: role of H-NS protein and the cyclic AMP-catabolite activator protein complex in transcription of the flhDC master operon. J Bacteriol 1999, 181(24):7500-7508.

5. Soutourina OA, Krin E, Laurent-Winter C, Hommais F, Danchin A, Bertin PN: Regulation of bacterial motility in response to low $\mathrm{pH}$ in Escherichia coli: the role of H-NS protein. Microbiology 2002, 148(5):1543-1551.

6. Krin E, Danchin A, Soutourina O: RcsB plays a central role in H-NSdependent regulation of motility and acid stress resistance in Escherichia coli. Res Microbiol 2010, 161(5):363-371.

7. Masuda N, Church GM: Regulatory network of acid resistance genes in Escherichia coli. Mol Microbiol 2003, 48(3):699-712.

8. Sayed AK, Odom C, Foster JW: The Escherichia coli AraC-family regulators GadX and GadW activate gadE, the central activator of glutamatedependent acid resistance. Microbiology 2007, 153(8):2584-2592.

9. Atlung T, Ingmer H: H-NS: a modulator of environmentally regulated gene expression. Mol Microbiol 1997, 24(1):7-17.

10. Hommais F, Krin E, Coppee JY, Lacroix C, Yeramian E, Danchin A, Bertin P: GadE (YhiE): a novel activator involved in the response to acid environment in Escherichia coli. Microbiology 2004, 150(1):61-72.

11. Weber H, Polen T, Heuveling J, Wendisch VF, Hengge R: Genome-wide analysis of the general stress response network in Escherichia coli: sigmaS-dependent genes, promoters, and sigma factor selectivity. J Bacteriol 2005, 187(5):1591-1603.

12. Shin $\mathrm{M}$, Song $\mathrm{M}$, Rhee JH, Hong $\mathrm{Y}$, Kim $\mathrm{YJ}$, Seok $\mathrm{YJ}$, Ha KS, Jung $\mathrm{SH}$, Choy HE: DNA looping-mediated repression by histone-like protein H-NS: specific requirement of Esigma70 as a cofactor for looping. Genes Dev 2005, 19(19):2388-2398.

13. Oshima T, Ishikawa S, Kurokawa K, Aiba H, Ogasawara N: Escherichia coli histone-like protein H-NS preferentially binds to horizontally acquired DNA in association with RNA polymerase. DNA Res 2006, 13(4):141-153.

14. Kieboom J, Abee T: Arginine-dependent acid resistance in Salmonella enterica serovar Typhimurium. J Bacteriol 2006, 188(15):5650-5653.

15. Stim-Herndon KP, Flores TM, Bennett GN: Molecular characterization of adiY, a regulatory gene which affects expression of the biodegradative acid-induced arginine decarboxylase gene (adiA) of Escherichia coli. Microbiology 1996, 142:1311-1320

16. Dell $\mathrm{CL}$, Neely $\mathrm{MN}$, Olson ER: Altered $\mathrm{pH}$ and lysine signalling mutants of cadC, a gene encoding a membrane-bound transcriptional activator of the Escherichia coli cadBA operon. Mol Microbiol 1994, 14(1):7-16.
17. Mechold U, Ogryzko V, Ngo S, Danchin A: Oligoribonuclease is a common downstream target of lithium-induced pAp accumulation in Escherichia coli and human cells. Nucleic Acids Res 2006, 34(8):2364-2373.

18. Baba T, Ara T, Hasegawa M, Takai Y, Okumura Y, Baba M, Datsenko KA, Tomita M, Wanner BL, Mori H: Construction of Escherichia coli K-12 inframe, single-gene knockout mutants: the Keio collection. Mol Syst Biol 2006, 2:2006 0008

19. Miki T, Yamamoto Y, Matsuda H: A novel, simple, high-throughput method for isolation of genome-wide transposon insertion mutants of Escherichia coli K-12. Methods Mol Biol 2008, 416:195-204.

20. Williams RM, Rimsky S, Buc H: Probing the structure, function, and interactions of the Escherichia coli H-NS and StpA proteins by using dominant negative derivatives. J Bacteriol 1996, 178(15):4335-4343

21. Goyard S, Bertin P: Characterization of $\mathrm{BpH} 3$, an H-NS-like protein in Bordetella pertussis. Mol Microbiol 1997, 24(4):815-823.

22. Giangrossi M, Zattoni S, Tramonti A, De Biase D, Falconi M: Antagonistic role of H-NS and GadX in the regulation of the glutamate decarboxylase-dependent acid resistance system in Escherichia coli. J Biol Chem 2005, 280(22):21498-21505.

23. Kuper $\mathrm{C}$, Jung $\mathrm{K}$ : CadC-mediated activation of the cadBA promoter in Escherichia coli. J Mol Microbiol Biotechnol 2005, 10(1):26-39.

24. Dorman CJ: H-NS, the genome sentinel. Nat Rev Microbiol 2007, 5(2):157-161.

25. Fang FC, Rimsky S: New insights into transcriptional regulation by H-NS Curr Opin Microbiol 2008, 11(2):113-120.

26. Malki A, Le HT, Milles S, Kern R, Caldas T, Abdallah J, Richarme G: Solubilization of protein aggregates by the acid stress chaperones HdeA and HdeB. J Biol Chem 2008, 283(20):13679-13687.

27. Waterman SR, Small PL: Identification of sigma S-dependent genes associated with the stationary-phase acid-resistance phenotype of Shigella flexneri. Mol Microbiol 1996, 21(5):925-940.

28. Rousseau F, Serrano L, Schymkowitz JW: How evolutionary pressure against protein aggregation shaped chaperone specificity. J Mol Biol 2006, 355(5):1037-1047.

29. Nair S, Finkel SE: Dps protects cells against multiple stresses during stationary phase. J Bacteriol 2004, 186(13):4192-4198.

30. Nakamura E, Torii K, Uneyama H: Physiological roles of dietary free glutamate in gastrointestinal functions. Biol Pharm Bull 2008, 31(10):1841-1843.

31. Gut H, Pennacchietti E, John RA, Bossa F, Capitani G, De Biase D, Grutter MG: Escherichia coli acid resistance: $\mathrm{pH}$-sensing, activation by chloride and autoinhibition in GadB. EMBO J 2006, 25(11): 2643-2651

32. Colonna B, Casalino M, Fradiani PA, Zagaglia C, Naitza S, Leoni L, Prosseda G, Coppo A, Ghelardini P, Nicoletti M: H-NS regulation of virulence gene expression in enteroinvasive Escherichia coli harboring the virulence plasmid integrated into the host chromosome. J Bacteriol 1995, 177(16):4703-4712.

33. Chen MH, Takeda S, Yamada H, Ishii $Y$, Yamashino T, Mizuno T: Characterization of the RcsC-> YojN- > RcsB phosphorelay signaling pathway involved in capsular synthesis in Escherichia coli. Biosci Biotechnol Biochem 2001, 65(10):2364-2367.

34. Grainger DC, Goldberg MD, Lee DJ, Busby SJ: Selective repression by Fis and H-NS at the Escherichia coli dps promoter. Mol Microbiol 2008, 68(6):1366-1377.

35. Itou J, Eguchi Y, Utsumi R: Molecular mechanism of transcriptional cascade initiated by the EvgS/EvgA system in Escherichia coli K-12. Biosci Biotechnol Biochem 2009, 73(4):870-878.

36. Ma Z, Richard H, Tucker DL, Conway T, Foster JW: Collaborative regulation of Escherichia coli glutamate-dependent acid resistance by two AraC-like regulators, GadX and GadW (YhiW). J Bacteriol 2002, 184(24):7001-7012.

37. Tramonti A, De Canio M, Delany I, Scarlato V, De Biase D: Mechanisms of transcription activation exerted by GadX and GadW at the gadA and gadBC gene promoters of the glutamate-based acid resistance system in Escherichia coli. J Bacteriol 2006, 188(23):8118-8127.

38. Tramonti A, Visca P, De Canio M, Falconi M, De Biase D: Functional characterization and regulation of $\mathrm{gadX}$, a gene encoding an AraC/XylSlike transcriptional activator of the Escherichia coli glutamic acid decarboxylase system. J Bacteriol 2002, 184(10):2603-2613. 
39. Tucker DL, Tucker N, Ma Z, Foster JW, Miranda RL, Cohen PS, Conway T: Genes of the GadX-GadW regulon in Escherichia coli. J Bacteriol 2003, 185(10):3190-3201.

40. Zhou Y, Gottesman S: Modes of regulation of RpoS by H-NS. J Bacteriol 2006, 188(19):7022-7025.

41. Neely MN, Dell CL, Olson ER: Roles of LysP and CadC in mediating the lysine requirement for acid induction of the Escherichia coli cad operon. J Bacteriol 1994, 176(11):3278-3285.

42. Bruni CB, Colantuoni V, Sbordone L, Cortese R, Blasi F: Biochemical and regulatory properties of Escherichia coli K-12 hisT mutants. J Bacteriol 1977, 130(1):4-10.

43. Bertin P, Benhabiles $N$, Krin E, Laurent-Winter $C$, Tendeng $C$, Turlin E, Thomas A, Danchin A, Brasseur R: The structural and functional organization of H-NS-like proteins is evolutionarily conserved in gramnegative bacteria. Mol Microbiol 1999, 31(1):319-329.

doi:10.1186/1471-2180-10-273

Cite this article as: Krin et al:: Decrypting the H-NS-dependent regulatory cascade of acid stress resistance in Escherichia coli. BMC Microbiology 2010 10:273.

\section{Submit your next manuscript to BioMed Central} and take full advantage of:

- Convenient online submission

- Thorough peer review

- No space constraints or color figure charges

- Immediate publication on acceptance

- Inclusion in PubMed, CAS, Scopus and Google Scholar

- Research which is freely available for redistribution

Submit your manuscript at www.biomedcentral.com/submit 